24 Evans MA, Bhat R, Vidyasagar D, Vadapalli M, Fisher E, Hastreiter A. Gestational age and indomethacin elimination in the neonate. Clin Pharmacol Ther 1979;26:746-51.

${ }^{25}$ Winther J, Printz MP, Mendoza SA, Kirkpatrick SE, Friedman WF The influence of indomethacin on neonatal renal function. Pediatr Re $1977 ; 11: 402$.

26 Yanagi RM, Wilson A, Newfeld EA, Aziz KU, Hunt CE. Indomethacin treatment for symptomatic patent ductus arteriosus: a double-blind control study. Pediatrics $1981 ; 67: 647-52$.

${ }^{27}$ Bejar R, Osorno L, Curbelo V, Schneider H, Coen R, Gluck L. Early PDA treatment with indomethacin (INDO) and intraventricula hemorrhage (IVH). Pediatr Res 1981;15:649.

\section{Removal of lymphocytes in rheumatoid arthritis}

Theories about the pathogenesis of rheumatoid arthritis are dominated by one central belief: that a continued response to unidentified antigens provokes inflammatory reactions that destroy the articular cartilage. A similar process is invoked with various other target organs in systemic autoimmune disorders. This dogma provides the basis for treating such diseases with cytotoxic drugs, and the benefits of drugs such as gold and penicillamine are generally attributed to their immunosuppressive actions.

As a logical extension of these views clinicians have looked for ways of affecting the recirculating pool of lymphocytes that initiates and sustains immune reactions. ${ }^{1}$ Firstly, thoracic duct drainage has been used (though not widely) in patients with rheumatoid arthritis and systemic erythematosus. ${ }^{23} \mathrm{Up}$ to $50.0 \times 10^{9}$ lymphocytes daily may be directly removed by this method; the considerable volume of lymphatic fluid also removed must be reinfused. Secondly, plasma exchange is being widely used to treat disorders thought to be mediated by immunological mechanisms, ${ }^{4}$ with the object of removing circulating immune complexes and inflammatory mediators. Cell separator machines are so designed, however, that lymphocytes may be deliberately removed at the same time, and the procedure can be modified to make this the primary purpose. In one study a mean of $31.0 \times 10^{9}$ lymphocytes was removed from four patients with rheumatoid arthritis over six weeks. ${ }^{5}$

Thirdly, lymphocytes may be inactivated by specific antisera-a method that proved very successful in suppressing experimental models of autoimmune disease. Transplant recipients excepted, however, antilymphocytic globulin has been used only sporadically in clinical practice.

Finally, selective $x$ irradiation of lymphoid tissues is receiving fresh attention for achieving prolonged immunosuppression. ${ }^{6}$ By carefully screening non-lymphoid organs, up to 4400 rads may be given in fractionated doses without incurring the formerly severe side effects of whole body irradiation. Studies in mice have shown that the lupus-like diseases of certain inbred strains can be suppressed by this method, which has also been reported as successful in a patient with severe rheumatoid arthritis. ${ }^{7}$

Clinical success has been claimed with all these methods. Drainage of the thoracic duct leads to an improvement in patients with rheumatoid arthritis, judging by such criteria as a reduction in the number of inflamed joints and improved functional capacity. ${ }^{2}$ The infiltration of rheumatoid synovial membrane by lymphocytes is also reduced. ${ }^{3}$ Severe cutaneous vasculitis resolved after thoracic duct drainage ${ }^{8}$ in a patient with systemic lupus erythematosus who had not responded to steroids and azathioprine. Clinical improvement seems to have been less impressive. "While reports of these techniques have been few, there have been numerous claims that plasma exchange benefits patients with connective tissue disease. Certainly this procedure seems to produce temporary improvement in diseases such as rheumatoid arthritis ${ }^{9}$ and systemic lupus erythematosus, ${ }^{10}$ but its long-term value remains problematical, given the dearth of properly controlled trials. ${ }^{11}$ Furthermore, these reports do not indicate that removal of lymphocytes has anything to do with the real or imagined benefits of plasma exchange.

The immunological consequences of these manoeuvres have been partially defined, particularly in patients subjected to thoracic duct drainage. ${ }^{23}$ The numbers of both $T$ and $B$ lymphocytes are reduced. Delayed hypersensitivity reactions are depressed, judged by prolonged survival of skin homografts and by responses to skin tests with standard antigens or dinitrochlorobenzene. Antibody responses after challenge with novel antigens such as keyhole-limpet haemocyanin or reimmunisation with tetanus toxoid are little affected. Nevertheless, the plasma concentrations of all the major immunoglobulin classes are reduced. Unfortunately such tests of immunological function have now become outdated.

What part these manoeuvres will play in the treatment of diseases such as rheumatoid arthritis cannot be predicted. As yet their therapeutic value has not been established. The immediate reduction in inflammatory activity is short lived, and is as much attributable to bed rest or other non-specific factors as to lymphocyte depletion. Spontaneous recovery of lymphocyte numbers or the reinfusion of lymphocytes removed by thoracic duct drainage ${ }^{2}$ leads to an exacerbation of disease activity. Thus these measures have not been shown to produce prolonged remissions. Most clinicians will doubt whether the cost, inconvenience, and hazards justify the temporary improvement to be expected in rheumatoid arthritis and systemic connective tissue diseases. Yet these disorders may still prove amenable to more refined methods of immunomanipulation.

Two approaches raise the possibility that such hopes will be fulfilled. Firstly, attempts at producing non-specific "lymphopenia" oversimplify the problem: improved methods of defining the immunological defects in these diseases are now being introduced. Secondly, the control of unwanted immune responses will eventually be attempted with monoclonal antisera directed at specific lymphocyte populations and not with non-specific immunosuppressive methods of the kind that have hitherto been used.

A M DenMan

Consultant Physician

Connective Tissue Diseases Research Group,

Clinical Research Centre,

London HAl $3 \mathrm{UJ}$

1 De Sousa M. Lymphocyte circulation: experimental and clinical aspects. Chichester: John Wiley, 1981

2 Paulus HE, Machleder HI, Clements PJ, Nyman K, Levine S. Prolonged thoracic-duct drainage in rheumatoid arthritis and systemic lupus erythematosus. West 7 Med 1979;130:309-24.

${ }^{3}$ Ueo T, Tanaka S, Tominaga Y, Ogawa H, Sakurami T. The effect of thoracic duct drainage on lymphocyte dynamics and clinical symptoms in patients with rheumatoid arthritis. Arthritis Rheum 1979;22:1405-12.

' Hamblin TJ. Plasmapheresis and plasma exchange. Vol 1. Edinburgh: Churchill Livingston, 1980. (Annual Research Reviews.)

5 Karsh J, Wright DG. Klippel JH, Decker JL, Deisseroth AB, Flye MW. Lymphocyte depletion by continuous flow cell centrifugation in rheumatoid arthritis. Arthritis Rheum 1979;22:1055-9.

6 Slavin S, Yatziv S, Zanbar I, Fuks Z, Kaplan HS, Strober S. Nonspecific and specific immunosuppression by total lymphoid irradiation (TLI). In: Fougereau $\mathrm{M}$, Dausset J, eds. Immunology 80. Vol 3. London: Academic Press, 1980:1160-70. (Progress in Immunology IV.) 
' Kotzin BL, Strober S, Calin A, Englemane E, Hoppe R, Kaplan HS The treatment of intractable rheumatoid arthritis with total lymphoid irradiation. Rev Rhum Mal Osteoartic $1981 ; \mathbf{4 8}$, special number, June: abst 0133 . (Proceedings of XV International Congress of Rheumatology.)

${ }^{*}$ Nyman KE, Bangert R, Machleder H, Paulus HE. Thoracic duct drainage in SLE with cutaneous vasculitis. A case report. Arthritis Rheum $1977 ; 20: 1129-34$.

${ }^{9}$ Wallace DJ, Goldfinger D, Gatti R, et al. Plasmapheresis and lymphoplasmapheresis in the management of rheumatoid arthritis. Arthritis Rheum $1979 ; 22: 703-10$

1" Parry HF, Moran CJ, Snaith ML, et al. Plasma exchange in systemic lupus erythematosus. Ann Rheum Dis 1981;40:224-8.

11 Anonymous. Plasma exchange. Ann Rheum Dis 1980;39:95-6.

\section{Histopathology reporting in large-bowel cancer}

The general objectives of medical audit are investigating standards of practice and improving the performance of individual practitioners. A good example of the success of audit in laboratory medicine has been the National Laboratory Quality Control Scheme, which in an initial study showed wide fluctuations in the performance of different biochemical departments in Britain. A later, repeat audit showed a substantial improvement by most of the participating laboratories. ${ }^{1}$ The hospital necropsy has proved its place in medical audit. ${ }^{2} 3$ Few reports of quality evaluation in diagnostic histopathology in Britain, either intradepartmental or interdepartmental, have been published, ${ }^{4}$ however, though Langley ${ }^{6}$ has emphasised the desirability of monitoring and improving diagnostic accuracy.

Just how histopathologists could benefit from more audit has now been shown very clearly by the report of the largebowel cancer project organised from St Mary's Hospital, London. It reviewed the histopathological reports on 2046 patients from 22 histopathology departments and found considerable observer variation in histological grading, Dukes's staging, and harvest of lymph nodes-all measures of established importance in the evaluation of prognosis after surgical treatment ${ }^{7}$ as well as in the management of patients and the design of operations. Some observer variation is to be expected in histological grading and that part of Dukes's staging concerned with estimation of the macroscopic extent of spread in operation specimens, because these are subjective observations. Even so, the observer variations on these two assessments went beyond what is acceptable. The harvesting of lymph nodes from operation specimens, on the other hand, is a purely objective method of examination; yet again large, and unexpected, differences were found in the mean numbers of lymph nodes removed per operation specimen in each hospital. Blenkinsopp and his colleagues ${ }^{8}$ point out that the most avoidable errors lay not in the assessment of microscopical appearances but in the gross or macroscopic observations. This suggests that the histopathologists concerned were not giving sufficient time or care to the "cut-up" and morbid anatomical examination of surgical specimens of large-bowel cancer.

If the reliability of Dukes's staging is poor, we need to ask whether histopathologists sufficiently appreciate the importance of accurately staging other varieties of malignant disease. For example, an interdepartmental audit of staging breast cancer, with emphasis on harvest of lymph nodes, might make interesting reading. Whether or not regional lymph nodes are affected is the single most important factor for prognosis and postoperative management in epithelial cancers of the bowel, breast, stomach, head and neck, and other sites. It is not enough to sample only the larger or obviously affected nodes, because only by dissection and microscopical examination of all regional nodes can the pathologist know whether any or how many are affected. The actual number of nodes affected is, indeed, a crucial objective observation of great value in prognosis.

In recent years interest in postmortem pathology in hospital practice has declined, and this could lead to a general devaluation of the importance of descriptive morbid anatomy. The report from the large-bowel cancer project suggests that histopathologists are not applying these descriptive skills to the careful evaluation of the detailed macroscopic appearances and to dissection of surgical material. Observations at this level can all too easily become cursory, especially when operation specimens are delivered to the laboratory formalin-fixed in an almost unrecognisable state. Here the responsibility lies partly with surgeons, who after all are the ones who should be most concerned with the quality of the information they receive. Histopathologists would give a better and more enthusiastic service if request forms always gave at least outline information about the surgical anatomy of an operation specimen, together with a formal request for the appropriate method of staging and grading. Unfortunately, form filling is most often delegated to junior staff (and sometimes to nursing staff), who give only sketchy information, if any, about operative findings. Failure by histopathologists to cultivate the skills of descriptive morbid anatomical examination of surgical specimens, whether in the form of the written word or by photographs and line drawings, could lead to the growth of "microscope pathologists," for whom the evaluation of the macroscopical appearance is a chore to be delegated to an unsupervised and inexperienced junior member of staff. Blenkinsopp and his colleagues point out that cancer of the large bowel is sufficiently common for consistent and reliable reports to be obtainable with relatively little effort: but that effort depends on co-operation between surgeon and pathologist.

The organisers of the large-bowel cancer project are to be congratulated on drawing attention to the need for more audit in diagnostic histopathology. The next stage-we hope-will be for them to repeat their work in a few years' time to see whether there has been any improvement in the performance of the 22 histopathology departments concerned. Staging and grading of cancer provides particularly important and valuable opportunities for medical audit because histopathologists, surgeons, radiotherapists, and medical oncologists are all equally implicated and concerned with the quality of the work. Epidemiologists, too, must have accurate documentation of data for the increasingly important part they play in studies of the detection and prevention of cancer. There is an opportunity here for the Royal College of Pathologists, which, by virtue of its responsibility for standards of work, is best fitted to initiate medical audit over a wide range of histopathological reporting. That much of such work is based on subjective observations makes the task more difficult than in clinical chemistry but no less desirable.

B C Morson

Consultant Pathologist,

St Mark's Hospital,

London EC1V 2PS

${ }^{1}$ Whitehead TP. Quality control in clinical chemistry. New York: J Wiley and Sons, 1977.

${ }^{2}$ Cameron HM, McGoogan E. A prospective study of 1152 hospital autopsies: I. Inaccuracies in death certification. $\mathcal{f}$ Pathol 1981;133: 273-83.

${ }^{3}$ Cameron HM, McGoogan E. A prospective study of 1152 hospital autopsies: II. Analysis of inaccuracies in clinical diagnoses and their significance. F Pathol $1981 ; \mathbf{1 3 3}: 285-300$. 